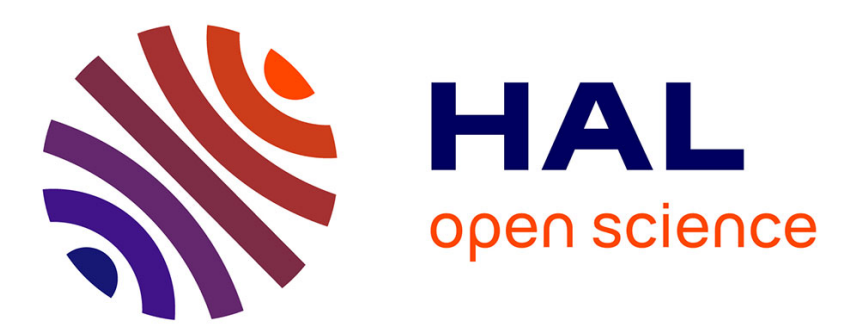

\title{
On the design of observer-based fuzzy adaptive controller for nonlinear systems with unknown control gain sign
}

\author{
Abdesselem Boulkroune, Mohammed M'Saad
}

\section{- To cite this version:}

Abdesselem Boulkroune, Mohammed M'Saad. On the design of observer-based fuzzy adaptive controller for nonlinear systems with unknown control gain sign. Fuzzy Sets and Systems, 2012, 201, pp.Pages 71-85. 10.1016/j.fss.2011.12.005 . hal-01061695

\section{HAL Id: hal-01061695 \\ https://hal.science/hal-01061695}

Submitted on 11 Sep 2014

HAL is a multi-disciplinary open access archive for the deposit and dissemination of scientific research documents, whether they are published or not. The documents may come from teaching and research institutions in France or abroad, or from public or private research centers.
L'archive ouverte pluridisciplinaire HAL, est destinée au dépôt et à la diffusion de documents scientifiques de niveau recherche, publiés ou non, émanant des établissements d'enseignement et de recherche français ou étrangers, des laboratoires publics ou privés. 


\title{
On the design of observer-based fuzzy adaptive controller for nonlinear systems with unknown control gain sign
}

\author{
A. Boulkroune ${ }^{\mathrm{a}, *}$, M. M'saad ${ }^{\mathrm{b}}$ \\ a Department of Automatic, Faculty of Engineering Sciences, University of Jijel, BP. 98, Ouled-Aissa, 18000 Jijel, Algeria \\ b GREYC, UMR 6072 CNRS, Université de Caen, ENSICAEN, 6 Bd Maréchal Juin, 14050 Caen Cedex, France
}

\begin{abstract}
In this paper, an observer-based fuzzy adaptive controller for nonlinear systems with unknown control gain sign is investigated. Because the system states are not available for measurement, a tracking-error observer is constructed. In this controller, the adaptive fuzzy system is used to approximate the unknown nonlinearities and the Nussbaum function is incorporated to deal with the unknown control direction (i.e. with the unknown control gain sign). The stability of the closed-loop system is proven using the strictly positive real (SPR) condition and Lyapunov theory. Finally, simulation results are given to verify the feasibility and effectiveness of the proposed controller.
\end{abstract}

Keywords: Fuzzy system; Adaptive control; Observer; Nussbaum function; SPR condition; Nonlinear system

\section{Introduction}

Fuzzy systems (FSs) have been successfully applied to many control problems because they do not need an accurate mathematical model of the system under control and they can co-operate with human expert knowledge. It is also known that FSs as well as neural networks (NNs) can approximate uniformly any nonlinear continuous function over a compact set $[12,29,30]$. Thanks to the universal approximation theorem [30], several adaptive fuzzy control schemes have been developed for uncertain nonlinear systems, e.g. $[6,8,25,26]$. The stability of the underlying control systems has been investigated using a Lyapunov approach. A key assumption in these control systems is that the state vector is assumed to be available for measurement. But this measurement requirement is more an exception than a rule in the engineering practice. That is why observer-based controllers (i.e. output-feedback controllers) are most used in practice.

Based on state or tracking-error observer, adaptive fuzzy control schemes have been developed in [14-16,27,31]. These schemes require strictly positive real (SPR) condition on the observation error dynamics (i.e. the estimation error dynamics) so that one can use Meyer-Kalman-Yakubovich (MKY) lemma in the stability analysis. The original observation error dynamics, which are not SPR in general, are augmented by a low-pass filter designed to satisfy the

\footnotetext{
* Corresponding author. Tel./fax: +21334501189 .

E-mail addresses: boulkroune2002@yahoo.fr (A. Boulkroune), msaad@greyc.ensicaen.fr (M. M'saad).
} 
SPR condition of a transfer function associated with the Lyapunov stability analysis. However, according to [20], these schemes result in the filtering of the fuzzy basis function (FBF) which makes the dynamic order of the controllerobserver system very large. Moreover, as stated in [1], these observer-based fuzzy adaptive controllers have not been derived rigorously in mathematics. In fact, some comments on these control schemes have been given in [1].

A common assumption in the schemes proposed in [1,14-17,20,27,31] is that the sign of the control gain is known a priori. However, in the general case, this assumption is by no means realistic as pointed out in [32]. When there is no a priori knowledge about the control gain sign, the design of the adaptive controllers based on observer for unknown nonlinear systems becomes more challenging. Especially, when the SPR condition is used in the control design and stability analysis. In this paper, unlike [1,14-17,20,27,31], an observer-based fuzzy adaptive controller is designed for a class of single-input single-output (SISO) nonlinear systems with unknown control gain sign. There are two main contributions that are worth to be emphasized:

- to the authors' best knowledge, there are little results reported on the fuzzy adaptive output-feedback control design based on SPR condition for nonlinear systems with unknown control gain sign [18]. Note that, in the design of fuzzy adaptive controller based on state observer, the combination of the Nussbaum-gain technique with the SPR condition is very difficult. This is why there are few results reported on this problem.

- unlike [14-17,27,31], in our proposed control law, there is no filtering of the FBF vector. Moreover, by using the SPR condition and Lyapunov theory, the stability of the closed-loop system is rigorously proven.

\section{Problem formulation and preliminaries}

In this paper, we consider the $n$th order nonlinear dynamical system of the form:

$$
\begin{aligned}
& x^{(n)}=f\left(x, \dot{x}, \ldots, x^{(n-1)}\right)+g\left(x, \dot{x}, \ldots, x^{(n-1)}\right) u+d(t), \\
& y=x .
\end{aligned}
$$

Or equivalently of the form

$$
\begin{aligned}
& \underline{\dot{x}}=A \underline{x}+B[f(\underline{x})+g(\underline{x}) u+d(t)], \\
& y=C^{T} \underline{x},
\end{aligned}
$$

where

$$
A=\left[\begin{array}{ccccc}
0 & 1 & 0 & \cdots & 0 \\
0 & 0 & 1 & \cdots & 0 \\
\vdots & \vdots & \vdots & \ddots & \vdots \\
0 & 0 & 0 & \cdots & 1 \\
0 & 0 & 0 & \cdots & 0
\end{array}\right], \quad B=\left[\begin{array}{c}
0 \\
0 \\
\vdots \\
0 \\
1
\end{array}\right], \quad C=\left[\begin{array}{c}
1 \\
0 \\
\vdots \\
0 \\
0
\end{array}\right],
$$

with $u \in R$ is the control input, $\underline{x}=\left[x, \dot{x}, \ldots, x^{(n-1)}\right]^{T}=\left[x_{1}, x_{2}, \ldots, x_{n}\right]^{T} \in R^{n}$ is the vector of unmeasured states and $y \in R$ is the measured output. $f(\underline{x})$ and $g(\underline{x})$ are unknown smooth functions, $d(t)$ is the external disturbance. Note that it is easy to verify that the pair $(A, B)$ is controllable and the pair $\left(C^{T}, A\right)$ is observable.

Our design objective consists in determining the control law $u$ to steer the system output $y$ closes to the reference signal $y_{r}$, while ensuring that involved signals in the closed-loop system remain bounded.

Assumption 1. There exists an unknown positive constant $d^{*}$ such that $|d(t)| \leq d^{*}$.

Assumption 2. The reference signals $y_{r}, \dot{y}_{r}, \ldots, y_{r}^{(n-1)}$, and $y_{r}^{(n)}$ are assumed to be continuous and bounded.

Assumption 3. The sign of the control gain $g(\underline{x})$ is also unknown. And there exist two unknown positive constants $g_{1}$ and $g_{0}$ such that: $0<g_{0} \leq|g(\underline{x})| \leq g_{1}[1,24]$. 
The following remarks allow to motivate the above assumptions with respect to the considered design framework:

Remark 1. Assumptions 1 is usually required in system theory, e.g. see [1,14,20,31]. Assumption 2 is a standard assumption in the adaptive control literature. This latter is the first to be made in an adaptive control scheme and can be given explicitly or implicitly.

Remark 2. Many practical systems can be expressed or transformed in the form (1) such as: inverted pendulum system, Duffing oscillator, Chua's chaotic circuit, mass-springer-damper system, aircraft wing rock, induction servo-motor, gyro system, Genesio chaotic system, single-link robot, and many others. Assumption 3 is not restrictive as it is satisfied by all these practical systems. It is worth noting that the property $0<g_{0} \leq|g(\underline{x})|$ guarantees the controllability of the system (1).

Let us define the reference signal vector $\underline{y}_{r}$ and the tracking error vector as follows:

$$
\underline{y}_{r}=\left[y_{r}, \dot{y}_{r}, \ldots, y_{r}^{(n-1)}\right]^{T}, \quad \underline{e}=\underline{y}_{r}-\underline{x}=\left[e, \dot{e}, \ldots, e^{(n-1)}\right]^{T}=\left[e_{1}, e_{2}, \ldots, e_{n}\right]^{T} .
$$

By using the fact that $\underline{\dot{y}}_{r}=A \underline{y}_{r}+B y_{r}^{(n)}$, we get

$$
\begin{aligned}
& \underline{\dot{e}}=A \underline{e}+B\left[y_{r}^{(n)}-f(\underline{x})-g(\underline{x}) u-d(t)\right], \\
& e_{1}=C^{T} \underline{e} .
\end{aligned}
$$

Based on the feedback linearization approach, when the functions $f(\underline{x})$ and $g(\underline{x})$ are known, $d(t)=0$ and the state $\underline{x}$ is available for measurement, the so-called ideal controller can be chosen as follows:

$$
u=u^{*}=g^{-1}(\underline{x})\left[-f(\underline{x})+y_{r}^{(n)}+K_{c}^{T} \underline{e}\right],
$$

where $K_{c}=\left[k_{c 1}, k_{c 2}, \ldots, k_{c n}\right]^{T} \in R^{n}$ is the feedback-gain vector selected such that the characteristic polynomial of $A-B K_{c}^{T}$ is strictly Hurwitz (i.e. stable).

Substituting (5) into (4) yields

$$
e^{(n)}+K_{c}^{T} \underline{e}=e^{(n)}+k_{c n} e^{(n-1)}+\cdots+k_{c 1} e=0 .
$$

Thus, it can be obtained that $\lim _{t \rightarrow \infty} e(t)=0$. However, since the functions $f(\underline{x})$ and $g(x)$ are unknown and the state vector $\underline{x}$ is not available for measurement, the ideal controller (5) cannot be implemented. Thereafter, to overcome such problems, we will use

- an adaptive fuzzy system to approximate the unknown nonlinear function,

- a Nussbaum function to estimate the sign of $g(\underline{x})$, and

- a state observer to estimate the tracking error vector.

\subsection{Description of the fuzzy logic system}

The basic configuration of a fuzzy logic system consists of a fuzzifier, some fuzzy IF-THEN rules, a fuzzy inference engine and a defuzzifier, as shown in Fig. 1.

The fuzzy inference engine uses the fuzzy IF-THEN rules to perform a mapping from an input vector $\underline{x}^{T}=\left[x_{1}, x_{2}, \ldots, x_{n}\right] \in R^{n}$ to an output $\hat{f} \in R$. The $i$ th fuzzy rule is written as

$$
R^{(i)}: \text { if } x_{1} \text { is } A_{1}^{i} \text { and } \ldots \text { and } x_{n} \text { is } A_{n}^{i} \text { then } \hat{f} \text { is } f^{i} \text {, }
$$

where $A_{1}^{i}, A_{2}^{i}, \ldots$, and $A_{n}^{i}$ are fuzzy sets and $f^{i}$ is the fuzzy singleton for the output in the $i$ th rule. By using the singleton fuzzifier, product inference, and center-average defuzzifier, the output of the fuzzy system can be expressed as follows:

$$
\hat{f}(\underline{x})=\frac{\sum_{i=1}^{m} f^{i}\left(\prod_{j=1}^{n} \mu_{A_{j}^{i}}\left(x_{j}\right)\right)}{\sum_{i=1}^{m}\left(\prod_{j=1}^{n} \mu_{A_{j}^{i}}\left(x_{j}\right)\right)}=\theta^{T} \psi(\underline{x}),
$$




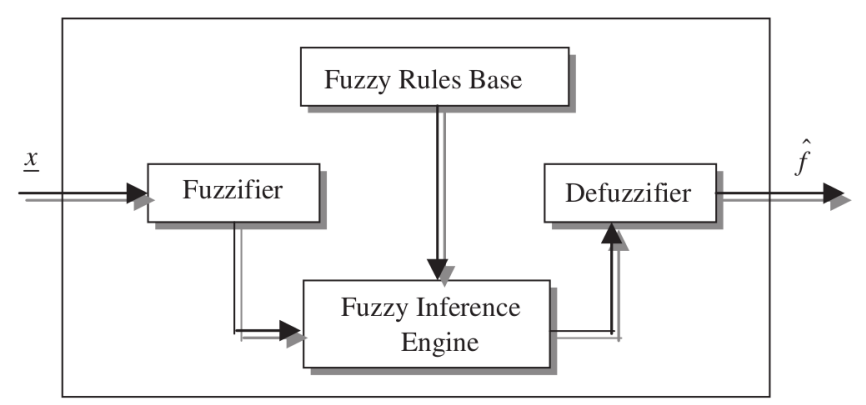

Fig. 1. The basic configuration of a fuzzy logic system.

where $\mu_{A_{j}^{i}}\left(x_{j}\right)$ is the degree of membership of $x_{j}$ to $A_{j}^{i}, m$ is the number of fuzzy rules, $\theta^{T}=\left[f^{1}, f^{2}, \ldots, f^{m}\right]$ is the adjustable parameter vector (composed of consequent parameters), and $\psi^{T}=\left[\begin{array}{llll}\psi^{1} & \psi^{2} \ldots \psi^{m}\end{array}\right]$ with

$$
\psi^{i}(\underline{x})=\frac{\left(\prod_{j=1}^{n} \mu_{A_{j}^{i}}\left(x_{j}\right)\right)}{\sum_{i=1}^{m}\left(\prod_{j=1}^{n} \mu_{A_{j}^{i}}\left(x_{j}\right)\right)}
$$

being the fuzzy basis function $(F B F)$. Throughout the paper, it is assumed that the FBFs are selected so that there is always at least one active rule [30], i.e. $\sum_{i=1}^{m}\left(\prod_{j=1}^{n} \mu_{A_{j}^{i}}\left(x_{j}\right)\right)>0$.

It is worth noting that the fuzzy system (7) is commonly used in control applications. Following the universal approximation results [30], the fuzzy system (7) is able to approximate any nonlinear smooth function $f(\underline{x})$ on a compact operating space to an arbitrary degree of accuracy. Of particular importance, it is assumed that the structure of the fuzzy system (i.e. the pertinent inputs, the number of membership functions for each input and the number of rules) and the membership function parameters are properly specified beforehand. The consequent parameters $\theta$ are then determined by appropriate adaptation algorithms.

\subsection{Nussbaum function}

In this paper, a Nussbaum function will be incorporated in the control law in order to estimate the sign of the control gain.

A function $N(\zeta)$ is called a Nussbaum-function, if it has the following useful properties $[10,19]$ :

(1) $\lim _{s \rightarrow+\infty} \sup (1 / s) \int_{0}^{s} N(\zeta) d \zeta=+\infty$,

(2) $\lim _{s \rightarrow+\infty} \inf (1 / s) \int_{0}^{s} N(\zeta) d \zeta=-\infty$.

Example. The following functions are Nussbaum functions [10]:

$$
\begin{aligned}
& N_{1}(\zeta)=\zeta^{2} \cos (\zeta), \\
& N_{2}(\zeta)=\cos ((\pi / 2) \zeta) e^{\zeta^{2}} .
\end{aligned}
$$

Of course, the cosine in the above examples can be replaced by the sine. It is very easy to see that $N_{1}(\zeta)$ and $N_{2}(\zeta)$ are Nussbaum functions.

As in [3,10], the even Nussbaum function $N(\zeta)=c \cos ((\pi / 2) \zeta) e^{\zeta^{2}}$ will be used throughout this paper. Physically, a Nussbaum-function can be visualized as a function of infinite gain and infinite switching frequency. The following lemma regarding with the property of Nussbaum function will be utilized in the control design and theorem proof of the next section. 
Lemma 1 (Ge et al. [10]). Let $V(\cdot)$ and $\zeta(\cdot)$ be smooth functions defined on $\left[0, t_{f}\right)$, with $V(t) \geq 0, \forall t \in\left[0, t_{f}\right)$, and $N(\cdot)$ be an even Nussbaum-type function. If the following inequality holds:

$$
V(t) \leq c_{0}+e^{-c_{1} t} \int_{0}^{t} g(\underline{x}(\tau)) N(\zeta) \dot{\zeta} e^{c_{1} \tau} d \tau+e^{-c_{1} t} \int_{0}^{t} \dot{\zeta} e^{c_{1} \tau} d \tau, \quad \forall t \in\left[0, t_{f}\right),
$$

where $c_{0}$ represents some suitable constant, $c_{1}$ is a positive constant, and $g(\underline{x}(\tau))$ is a time-varying parameter which takes values in the unknown closed interval $I=\left[l^{-}, l^{+}\right]$, with $0 \notin I$, then $V(t), \zeta(t)$ and $\int_{0}^{t} g(\underline{x}(\tau)) N(\zeta) \dot{\zeta} d \tau$ must be bounded on $\left[0, t_{f}\right)$.

Proof of Lemma 1. To proof this lemma, see [10].

Remark 3. As pointed out in [11], Lemma 1 is true for all the Nussbaum functions. Because of the presence of $e^{c_{1} \tau}$ in (8), the proof is function-dependent. In addition, we underline that $N(\cdot)$ is not necessarily to be an even function, which is only made for convenience of the proof. If $N(\cdot)$ is chosen as an odd function, e.g. $N(\zeta)=\zeta^{2} \sin (\zeta)$, the lemma can be easily proven by following the same procedure in [10].

Lemma 1 can be easily extended to case where $t_{f}=\infty$ due to Proposition 1 given below. Consider [11]:

$$
\dot{x}(t)=F(x(t)) \quad \text { with } x(0)=x_{0},
$$

where $z \mapsto F(z) \in R^{N}$ is upper semicontinuous on $R^{n}$ with non-empty convex and compact values. It is well known that the initial-value problem has a solution and that every solution can be maximally extended.

Proposition 1 (Ryan [22]). If $x:\left[0, t_{f}\right) \rightarrow R^{N}$ is a bounded maximal solution of $(9)$, then $t_{f}=\infty$.

\section{The design of the observer-based fuzzy adaptive controller}

Consider now the following observer for estimating the tracking error vector $\underline{e}$ :

$$
\begin{aligned}
& \underline{\dot{\hat{e}}}=A_{c} \underline{\hat{e}}+K_{o} \tilde{e}_{1}, \\
& \hat{e}_{1}=C^{T} \underline{\hat{e}},
\end{aligned}
$$

where $A_{c}=A-B K_{c}^{T}, \tilde{e}_{1}=e_{1}-\hat{e}_{1}=\hat{y}-y, \underline{\hat{e}}=\underline{\hat{x}}-\underline{y}_{r}$, with $\underline{\hat{x}}$ is the estimate of the state vector $\underline{x}$ and $\underline{\hat{e}}$ is the estimate of the tracking error vector $\underline{e} . K_{o}=\left[k_{o 1}, k_{o 2}, \ldots, k_{o n}\right]^{T} \in R^{n}$ is the observer-gain vector to be selected such that the characteristic polynomial of $A_{c}-K_{o} C^{T}$ is strictly Hurwitz and the vector $K_{c}$ has been previously defined.

Let us define the observation error vector as $\underline{\tilde{e}}=\underline{e}-\underline{\hat{e}}=\left[\tilde{e}_{1}, \tilde{e}_{2}, \ldots, \tilde{e}_{n}\right]^{T}$. Subtracting (10) from (4), we get the dynamics of the observation error as

$$
\begin{aligned}
& \underline{\dot{\tilde{e}}}=A_{o} \underline{\tilde{e}}+B\left[f_{1}\left(\underline{x}_{v}\right)-g(\underline{x}) u-d(t)\right], \\
& \tilde{e}_{1}=C^{T} \underline{\tilde{e}}
\end{aligned}
$$

with $A_{o}=A_{c}-K_{o} C^{T}, f_{1}\left(\underline{x}_{v}\right)=f(\underline{x})+v$, where $\underline{x}_{v}=\left[x^{T}, v\right]^{T}$ and $v=y_{r}^{(n)}+K_{c}^{T} \underline{e}$.

The unknown continuous nonlinear function $f_{1}\left(\underline{x}_{v}\right)$ can be approximated, on the compact set $\Omega_{\underline{x}}$, by the fuzzy system (7) as follows:

$$
\hat{f}_{1}\left(\underline{x}_{v}, \theta\right)=\theta^{T} \psi\left(\underline{x}_{v}\right),
$$

where $\psi\left(\underline{x}_{v}\right)$ is the FBF vector fixed a priori by the designer and $\theta$ is the adjustable parameter vector of the fuzzy system.

Let us define the optimal value of $\theta$ as follows:

$$
\theta^{*}=\underset{\theta}{\arg \min }\left[\sup _{x \in \Omega_{\underline{x}}}\left|f_{1}\left(\underline{x}_{v}\right)-\hat{f}_{1}\left(\underline{x}_{v}, \theta\right)\right|\right] \text {. }
$$


Notice that the optimal value of $\theta$ (i.e. $\theta^{*}$ ) is introduced only for analysis purposes, and its value is not needed when implementing the controller.

Define

$$
\tilde{\theta}=\theta-\theta^{*}
$$

as the parameter estimation error, and

$$
\delta_{0}\left(\underline{x}_{v}\right)=f_{1}\left(\underline{x}_{v}\right)-\hat{f}_{1}\left(\underline{x}_{v}, \theta^{*}\right)
$$

as the fuzzy approximation error, where $\hat{f}_{1}\left(\underline{x}_{v}, \theta^{*}\right)=\theta^{* T} \psi\left(\underline{x}_{v}\right)$.

As in the literature [1-5,14-17,20,27,28,31], we assume that the used fuzzy system does not violate the universal approximator property on the compact set $\Omega_{\underline{x}}$. The latter is assumed to be large enough so that the input vector of the fuzzy system remains in $\Omega_{\underline{x}}$ under the closed-loop control system. So it is reasonable to assume that the fuzzy approximation error is bounded for all $x \in \Omega_{\underline{x}}$, i.e.

$$
\left|\delta_{0}\left(\underline{x}_{v}\right)\right| \leq \bar{\delta}_{0}, \quad \forall x \in \Omega_{\underline{x}},
$$

where $\bar{\delta}_{0}$ is an unknown constant.

Since the input vector $\underline{x}_{v}=\left[x^{T}, v\right]^{T}$ is not available for measurement, the fuzzy system (12) used to approximate $f_{1}\left(\underline{x}_{v}\right)$ is replaced by the following fuzzy system:

$$
\hat{f}_{1}\left(\underline{\hat{x}}_{v}, \theta\right)=\theta^{T} \psi\left(\underline{\hat{x}}_{v}\right),
$$

where the vector $\underline{\hat{x}}_{v}=\left[\hat{x}^{T}, \hat{v}\right]^{T}=\left[\hat{x}^{T}, K_{c}^{T} \underline{\hat{e}}+y_{r}^{(n)}\right]^{T}$ is the estimate of $\underline{x}_{v}=\left[x^{T}, v\right]^{T}$.

From (14)-(16), we have

$$
\begin{aligned}
f_{1}\left(\underline{x}_{v}\right) & =f_{1}\left(\underline{x}_{v}\right)-\hat{f}_{1}\left(\underline{x}_{v}, \theta^{*}\right)+\hat{f}_{1}\left(\underline{x}_{v}, \theta^{*}\right)-\hat{f}_{1}\left(\underline{\hat{x}}_{v}, \theta^{*}\right)+\hat{f}_{1}\left(\underline{\hat{x}}_{v}, \theta^{*}\right) \\
& =\hat{f}_{1}\left(\underline{\hat{x}}_{v}, \theta^{*}\right)+f_{1}\left(\underline{x}_{v}\right)-\hat{f}_{1}\left(\underline{x}_{v}, \theta^{*}\right)+\hat{f}_{1}\left(\underline{x}_{v}, \theta^{*}\right)-\hat{f}_{1}\left(\underline{\hat{x}}_{v}, \theta^{*}\right) \\
& =\theta^{* T} \psi\left(\underline{\hat{x}}_{v}\right)+\delta_{0}\left(\underline{x}_{v}\right)+\left[\theta^{* T} \psi\left(\underline{x}_{v}\right)-\theta^{* T} \psi\left(\underline{\hat{x}}_{v}\right)\right] \\
& =\theta^{* T} \psi\left(\underline{\hat{x}}_{v}\right)+\delta_{1}\left(\underline{x}_{v}, \underline{\hat{x}}_{v}\right),
\end{aligned}
$$

where $\delta_{1}\left(\underline{x}_{v}, \underline{\hat{x}}_{v}\right)=\delta_{0}\left(\underline{x}_{v}\right)+\left[\theta^{* T} \psi\left(\underline{x}_{v}\right)-\theta^{* T} \psi\left(\underline{\hat{x}}_{v}\right)\right]$ is the approximation error. Notice that $\delta_{1}\left(\underline{\hat{x}}_{v}, \underline{x}_{v}\right)$ has an upper bound, i.e. $\left|\delta_{1}\left(\underline{x}_{v}, \underline{\hat{x}}_{v}\right)\right| \leq \bar{\delta}_{1}$ with $\bar{\delta}_{1}$ is an unknown positive constant $[1,14-17,20,27,31]$.

Substituting (17) into (11) yields

$$
\begin{aligned}
& \underline{\dot{\tilde{e}}}=A_{o} \underline{\tilde{\tilde{e}}}+B\left[\theta^{* T} \psi\left(\underline{\hat{x}}_{v}\right)-g(\underline{x}) u+\delta_{2}\right], \\
& \tilde{e}_{1}=C^{T} \underline{\underline{\tilde{e}}}
\end{aligned}
$$

where $\delta_{2}=\delta_{1}\left(\underline{x}_{v}, \underline{\hat{x}}_{v}\right)-d(t)$.

Since $\delta_{1}\left(\underline{x}_{v}, \underline{\hat{x}}_{v}\right)$ and $d(t) \in L_{\infty}$, thus the upper bound of $\delta_{2}$ also exists, i.e. $\left|\delta_{2}\right| \leq \bar{\delta}_{2}$, with $\bar{\delta}_{2}$ is an unknown positive constant.

The dynamics (18) can be expressed in frequency domain using the mixed notation (i.e. time-frequency) which is very common in the adaptive control literature such as in $[9,13,23,24]$ :

$$
\tilde{e}_{1}=H(s)\left[\theta^{* T} \psi\left(\hat{x}_{v}\right)-g(\underline{x}) u+\delta_{2}\right],
$$

where $s$ is the Laplace variable and $H(s)=C^{T}\left(s I-A_{o}\right)^{-1} B$ is the stable transfer function of (18).

Note that the mixed notation in (19) refers to the convolution between the inverse Laplace transform of $H(s)$ and $\left[\theta^{* T} \psi\left(\underline{\hat{x}}_{v}\right)-g(\underline{x}) u+\delta_{2}\right]$. 
Now, since the observation error dynamics (19) are not SPR, we introduce a low pass filter $T(s)$ such that $\bar{H}(s)=H(s) T^{-1}(s)$ is SPR:

$$
\begin{aligned}
\tilde{e}_{1} & =H(s) T^{-1}(s)\left(T(s)\left[\theta^{* T} \psi\left(\underline{\hat{x}}_{v}\right)\right]-T(s)[g(\underline{x}) u]+T(s)\left[\delta_{2}\right]\right) \\
& =\bar{H}(s)\left(\theta^{* T} \psi_{f}\left(\underline{\hat{x}}_{v}\right)-T(s)[g(\underline{x}) u]+\delta_{2 f}\right) \\
& =\bar{H}(s)\left(\theta^{* T} \psi\left(\underline{\hat{x}}_{v}\right)-T(s)[g(\underline{x}) u]+\delta_{3}\right),
\end{aligned}
$$

where

$$
\begin{aligned}
& \psi_{f}\left(\underline{\hat{x}}_{v}\right)=T(s)\left[\psi\left(\underline{\hat{x}}_{v}\right)\right], \\
& \theta^{* T} \psi_{f}\left(\underline{\hat{x}}_{v}\right)=T(s)\left[\theta^{* T} \psi\left(\underline{\hat{x}}_{v}\right)\right]=\theta^{* T} T(s)\left[\psi\left(\underline{\hat{x}}_{v}\right)\right], \\
& \delta_{2 f}=T(s)\left[\delta_{2}\right], \\
& \delta_{3}=\theta^{* T}\left[\psi_{f}\left(\underline{\hat{x}}_{v}\right)-\psi\left(\underline{\hat{x}}_{v}\right)\right]+\delta_{2 f},
\end{aligned}
$$

and $T(s)$ is called a SPR filter.

Because $\delta_{2}, \psi\left(\underline{\hat{x}}_{v}\right)$ and $\theta^{*} \in L_{\infty}$ and $T(s)$ is a stable filter, therefore the term $\delta_{3}$ can be also bounded as follows: $\left|\delta_{3}\right| \leq \bar{\delta}_{3}$, where $\bar{\delta}_{3}$ is an unknown positive constant.

From (20), it is clear that the presence of the filtered term $T(s)[g(x) u]$ in the output observation-error dynamics makes the control system design very difficult. To facilitate this task, the following assumption is made.

Assumption 4. The following inequality holds:

$$
|g(\underline{x}) u-T(s)[g(\underline{x}) u]| \leq \lambda^{*}\left|u-u_{f}\right|,
$$

where $\lambda^{*}$ is an unknown positive constant and $u_{f}=T(s)[u]$.

Remark 4. For a general class of nonlinear systems, Assumption 4 can be relatively restrictive. However, it is already satisfied in the following special cases:

- if $g(\underline{x})$ is constant, and

- if $g(\underline{x})$ varies slowly, i.e. in the case where $T(s)[g(\underline{x}) u] \approx g(\underline{x}) T(s)[u]$.

Note that there exist many practical systems satisfying Assumption 4, e.g. mass-springer-damper system, aircraft wing rock, induction servo-motor, gyro system, single-link robot, and many others.

The control input for the system (1) can be determined as

$$
u=N(\xi)\left(-\theta^{T} \psi\left(\underline{\hat{x}}_{v}\right)-\rho \tanh \left(e_{m 1} / \varepsilon_{1}\right)\right),
$$

with

$$
\begin{aligned}
& N(\zeta)=\cos ((\pi / 2) \zeta) e^{\zeta^{2}} \\
& \dot{\zeta}=e_{m 1}\left(\theta^{T} \psi\left(\underline{\hat{x}}_{v}\right)+\rho \tanh \left(e_{m 1} / \varepsilon_{1}\right)\right) \\
& \dot{\theta}=-\gamma_{1} \sigma_{1} \theta+\gamma_{1} e_{m 1} \psi\left(\underline{\hat{x}}_{v}\right) \\
& \dot{\rho}=-\gamma_{2} \sigma_{2} \rho+\gamma_{2} e_{m 1} \tanh \left(e_{m 1} / \varepsilon_{1}\right),
\end{aligned}
$$

where $\gamma_{1}, \gamma_{2}, \sigma_{1}, \sigma_{2}$ and $\varepsilon_{1}$ are positive design constants, $\rho$ is the estimate of $\rho^{*}=\bar{\delta}_{3}$, and $\tanh (\cdot)$ denotes the hyperbolic tangent function. A new error $e_{m 1}$, called the modified error, is defined by

$$
e_{m 1}=\tilde{e}_{1}+e_{a 1}
$$


where the error $e_{a 1}$ is called the auxiliary error. It is generated by the following dynamics:

$$
e_{a 1}=\bar{H}(s)\left(-\lambda\left(u-u_{f}\right) \tanh \left(\left(u-u_{f}\right) e_{m 1} / \varepsilon_{2}\right)\right),
$$

with

$$
\dot{\lambda}=-\gamma_{3} \sigma_{3} \lambda+\gamma_{3} e_{m 1}\left(u-u_{f}\right) \tanh \left(\left(u-u_{f}\right) e_{m 1} / \varepsilon_{2}\right),
$$

where $\gamma_{3}, \sigma_{3}$ and $\varepsilon_{2}$ are positive design constants and $\lambda$ is the estimate of $\lambda^{*}$.

From (20), (27) and (28), the dynamics of the modified error $e_{m 1}$ can be expressed as follows:

$$
\begin{aligned}
e_{m 1} & =\bar{H}(s)\left(\theta^{* T} \psi\left(\underline{\hat{x}}_{v}\right)-T(s)[g(\underline{x}) u]-\lambda\left(u-u_{f}\right) \tanh \left(\left(u-u_{f}\right) e_{m 1} / \varepsilon_{2}\right)+\delta_{3}\right) \\
& =\bar{H}(s)\left(\theta^{* T} \psi\left(\underline{\hat{x}}_{v}\right)-g(\underline{x}) u+\Delta_{u}+\delta_{3}\right),
\end{aligned}
$$

where $\Delta_{u}=g(\underline{x}) u-T(s)[g(\underline{x}) u]-\lambda\left(u-u_{f}\right) \tanh \left(\left(u-u_{f}\right) e_{m 1} / \varepsilon_{2}\right)$.

Substituting (22) into (30) yields

$$
\begin{aligned}
e_{m 1} & =\bar{H}(s)\left(\theta^{* T} \psi\left(\hat{\underline{x}}_{v}\right)-g(\underline{x}) u+\Delta_{u}+\delta_{3}\right) \\
& =\bar{H}(s)\left(-\tilde{\theta}^{T} \psi\left(\underline{\hat{x}}_{v}\right)-\rho \tanh \left(e_{m 1} / \varepsilon_{1}\right)+(1+g(\underline{x}) N(\zeta))\left(\theta^{T} \psi\left(\underline{\hat{x}}_{v}\right)+\rho \tanh \left(e_{m 1} / \varepsilon_{1}\right)\right)+\Delta_{u}+\delta_{3}\right),
\end{aligned}
$$

where $\tilde{\theta}=\theta-\theta^{*}$.

The state space realization of (31) is given by

$$
\begin{aligned}
& \underline{\dot{e}}_{m}=\bar{A}_{o} \underline{\underline{e}}_{m}+\bar{B}\left[-\tilde{\theta}^{T} \psi\left(\underline{\hat{x}}_{v}\right)-\rho \tanh \left(e_{m 1} / \varepsilon_{1}\right)+(1+g(\underline{x}) N(\zeta))\left(\theta^{T} \psi\left(\underline{\hat{x}}_{v}\right)+\rho \tanh \left(e_{m 1} / \varepsilon_{1}\right)\right)+\Delta_{u}+\delta_{3}\right], \\
& e_{m 1}=\bar{C}^{T} \underline{\boldsymbol{e}}_{m},
\end{aligned}
$$

where $\underline{e}_{m}=\left[e_{m 1}, e_{m 2}, \ldots, e_{m n}\right]^{T}$ and $\left(\bar{A}_{o} \in R^{n \times n}, \bar{B} \in R^{n}, \bar{C} \in R^{n}\right)$ is a minimal state realization of

$$
\bar{H}(s)=H(s) T^{-1}(s)=\bar{C}^{T}\left(s I-\bar{A}_{o}\right)^{-1} \bar{B},
$$

with $\bar{C}=[0, \ldots, 0] 1^{T}$.

Since $\bar{H}(s)$ is SPR, the following holds:

$$
\begin{aligned}
& \bar{A}_{o}^{T} P+P \bar{A}_{o}=-Q<0, \\
& P \bar{B}=\alpha \bar{C},
\end{aligned}
$$

where $\alpha$ is a positive design constant, $P=P^{T}>0$ and $Q=Q^{T}>0$. Note that the matrix equations (33) and the dynamics (32) will be used in the stability analysis.

To summarize, Fig. 2 shows the scheme of the proposed fuzzy adaptive controller.

Theorem 1. Consider the system (1) under Assumptions 1-4 and the observer (10). Then, the proposed fuzzy adaptive controller, defined by (22)-(29), guarantees the following properties:

1. All signals in the closed-loop system are bounded, i.e. $\underline{e}_{m}, e_{m 1}, e_{a 1}, \tilde{e}_{1}, \underline{\tilde{e}}, \underline{\hat{e}}, \underline{e}, \theta$ and $u \in L_{\infty}$.

2. The output tracking error remains in a compact set $\Omega_{e}$ specified as: $\Omega_{e}=\left\{e_{1} \| e_{1} \mid \leq \kappa\right\}$, where $\kappa$ is a positive constant which will be defined later.

Proof of Theorem 1. Let us consider the Lyapunov function candidate

$$
V=\frac{1}{2 \alpha} \underline{e}_{m}^{T} P \underline{e}_{m}+\frac{1}{2 \gamma_{1}} \tilde{\theta}^{T} \tilde{\theta}+\frac{1}{2 \gamma_{2}} \tilde{\rho}^{2}+\frac{1}{2 \gamma_{3}} \tilde{\lambda}^{2},
$$

where $\tilde{\theta}=\theta-\theta^{*}$. 


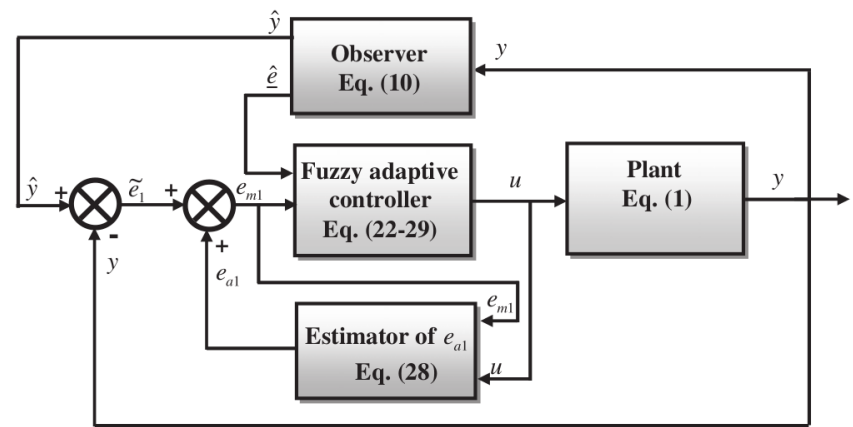

Fig. 2. The scheme of the proposed adaptive fuzzy controller based on observer.

Using (32) and (33), the time derivative of $V_{1}$ becomes

$$
\begin{aligned}
\dot{V}= & -\frac{1}{2 \alpha} \underline{e}_{m}^{T} Q \underline{e}_{m}+e_{m 1}\left[-\tilde{\theta}^{T} \psi\left(\underline{\hat{x}}_{v}\right)-\rho \tanh \left(e_{m 1} / \varepsilon_{1}\right)+(1+g(\underline{x}) N(\zeta))\left(\theta^{T} \psi\left(\underline{x}_{v}\right)+\rho \tanh \left(e_{m 1} / \varepsilon_{1}\right)\right)\right. \\
& \left.+\Delta_{u}+\delta_{3}\right]+\frac{1}{\gamma_{1}} \tilde{\theta}^{T} \dot{\theta}+\frac{1}{\gamma_{2}} \tilde{\rho} \dot{\rho}+\frac{1}{\gamma_{3}} \tilde{\lambda} \dot{\lambda} .
\end{aligned}
$$

Evaluating (35) along the trajectories (24) and (25) gives

$$
\dot{V}=-\frac{1}{2 \alpha} \underline{e}_{m}^{T} Q_{\underline{e}_{m}}+(1+g(\underline{x}) N(\zeta)) \dot{\zeta}-\sigma_{1} \tilde{\theta}^{T} \theta+e_{m 1}\left[-\rho \tanh \left(e_{m 1} / \varepsilon_{1}\right)+\delta_{3}+\Delta_{u}\right]+\frac{1}{\gamma_{2}} \tilde{\rho} \dot{\rho}+\frac{1}{\gamma_{3}} \tilde{\lambda} \dot{\lambda} .
$$

Using the following nice property with regard to function $\tanh (\cdot)[21]$ :

$$
0 \leq|z|-z \tanh (z / \varepsilon) \leq \bar{\varepsilon}=0.2785 \varepsilon,
$$

we can obtain the following inequalities:

$$
\begin{aligned}
e_{m 1} \delta_{3}-\rho e_{m 1} \tanh \left(e_{m 1} / \varepsilon_{1}\right) & \leq \rho^{*}\left|e_{m 1}\right|-\rho e_{m 1} \tanh \left(e_{m 1} / \varepsilon_{1}\right) \\
& \leq \rho^{*} \bar{\varepsilon}_{1}-\tilde{\rho} e_{m 1} \tanh \left(e_{m 1} / \varepsilon_{1}\right), \\
e_{m 1} \Delta_{u} & \leq \lambda^{*}\left|u-u_{f}\right|\left|e_{m 1}\right|-\lambda\left(u-u_{f}\right) e_{m 1} \tanh \left(\left(u-u_{f}\right) e_{m 1} / \varepsilon_{2}\right) \\
& \leq \lambda^{*} \bar{\varepsilon}_{2}-\tilde{\lambda}\left(u-u_{f}\right) e_{m 1} \tanh \left(\left(u-u_{f}\right) e_{m 1} / \varepsilon_{2}\right),
\end{aligned}
$$

where $\bar{\varepsilon}_{1}=0.2785 \varepsilon_{1}$ and $\bar{\varepsilon}_{2}=0.2785 \varepsilon_{2}$.

From (26), (29), (37) and (38), we can rewrite (36) as follows:

$$
\dot{V} \leq-\frac{1}{2 \alpha} \underline{e}_{m}^{T} Q \underline{e}_{m}+(1+g(\underline{x}) N(\zeta)) \dot{\zeta}-\sigma_{1} \tilde{\theta}^{T} \theta-\sigma_{2} \tilde{\rho} \rho-\sigma_{3} \tilde{\lambda} \lambda+\lambda^{*} \bar{\varepsilon}_{2}+\rho^{*} \bar{\varepsilon}_{1} .
$$

Since the following inequalities are valid:

$$
\begin{aligned}
& -\sigma_{1} \tilde{\theta}^{T} \theta \leq-\frac{\sigma_{1}}{2}\|\tilde{\theta}\|^{2}+\frac{\sigma_{1}}{2}\left\|\theta^{*}\right\|^{2}, \\
& -\sigma_{2} \tilde{\rho} \rho \leq-\frac{\sigma_{2}}{2} \tilde{\rho}^{2}+\frac{\sigma_{2}}{2} \rho^{* 2},
\end{aligned}
$$

and

$$
-\sigma_{3} \tilde{\lambda} \lambda \leq-\frac{\sigma_{3}}{2} \tilde{\lambda}^{2}+\frac{\sigma_{3}}{2} \lambda^{* 2}
$$

we can rewrite (39) as follows:

$$
\dot{V} \leq-\frac{1}{2 \alpha} \underline{e}_{m}^{T} Q \underline{e}_{m}-\frac{\sigma_{1}}{2}\|\tilde{\theta}\|^{2}-\frac{\sigma_{2}}{2} \tilde{\rho}^{2}-\frac{\sigma_{3}}{2} \tilde{\lambda}^{2}+\pi+(1+g(\underline{x}) N(\tilde{\zeta})) \dot{\zeta},
$$

where $\pi=\left(\sigma_{1} / 2\right)\left\|\theta^{*}\right\|^{2}+\left(\sigma_{2} / 2\right) \rho^{* 2}+\left(\sigma_{3} / 2\right) \lambda^{* 2}+\lambda^{*} \bar{\varepsilon}_{2}+\rho^{*} \bar{\varepsilon}_{1}$. 
Let $\mu=\min \left\{\lambda_{\min }(Q) / \lambda_{\max }(P), \gamma_{1} \sigma_{1}, \gamma_{2} \sigma_{2}, \gamma_{3} \sigma_{3}\right\}$, hence we can rewrite (40) as follows:

$$
\dot{V} \leq-\mu V+\pi+(1+g(\underline{x}) N(\zeta)) \dot{\zeta},
$$

where $\lambda_{\min }(Q)$ denotes the smallest eigen-value of $Q$, and $\lambda_{\max }(P)$ the largest eigen-value of $P$.

Multiplying (41) by $e^{\mu t}$ yields

$$
\frac{d}{d t}\left(V e^{\mu t}\right) \leq \pi e^{\mu t}+e^{\mu t}(1+g(\underline{x}) N(\zeta)) \dot{\zeta}
$$

Integrating (42) over $[0, t]$, it follows that

$$
0 \leq V(t) \leq \frac{\pi}{\mu}+\left(V(0)-\frac{\pi}{\mu}\right) e^{-\mu t}+e^{-\mu t} \int_{0}^{t}[(1+g(\underline{x}) N(\zeta))] e^{\mu \tau} \dot{\zeta} d \tau
$$

We can rewrite (43) as follows:

$$
0 \leq V(t) \leq \eta+e^{-\mu t} \int_{0}^{t}[(1+g(\underline{x}) N(\zeta))] e^{\mu \tau} \dot{\zeta} d \tau,
$$

where $\eta=\pi / \mu+V(0)$.

According to Lemma 1, we can conclude from (44) that $V(t), \zeta(t)$ and $\int_{0}^{t}[(1+g(\underline{x}) N(\zeta))] \dot{\zeta} d \tau$ are all bounded on $\left[0, t_{f}\right)$. The boundedness of $\theta, \rho, \lambda$ and $\underline{e}_{m}$ on $\left[0, t_{f}\right)$ follows that of $V(t)$. From the boundedness of $\theta, \rho$ and $\zeta$, we can conclude directly about the boundedness of $u$. Since $u \in L_{\infty}$ and $T(s)$ is a stable filter, then $u_{f} \in L_{\infty}$. From (18), since the term $\left[\theta^{* T} \psi\left(\underline{\hat{x}}_{v}\right)-g(\underline{x}) u+\delta_{2}\right]$ is bounded and $A_{o}$ is stable, we can easily show that the observation error vector $\underline{\tilde{e}}$ is also bounded. Because $e_{a 1}=e_{m 1}-\tilde{e}_{1}$, from the boundedness of $\tilde{e}_{1}$ and $e_{m 1}$, we can conclude directly the boundedness of $e_{a 1}$. From (10), the boundedness of $\underline{\hat{e}}$ follows that of $\tilde{e}_{1}$. Since $\underline{\tilde{e}}$ and $\underline{\hat{e}}$ are bounded, then the tracking error vector $\underline{e}$ is also bounded (i.e. $\underline{e} \in L_{\infty}$ ).

Furthermore, owing to the smoothness of the proposed controller, the closed-loop system admits a solution on its maximum interval of existence $\left[0, t_{f}\right.$ ). Therefore, according to Proposition 1 , no finite time escape phenomenon may occur and thus $t_{f}$ can be extended to $\infty[10,22]$. As an immediate result, all signals in the closed-loop system are bounded on $[0, \infty)$.

Let $\left|e^{-\mu t} \int_{0}^{t}[(1+g(\underline{x}) N(\zeta))] \dot{\zeta} e^{\mu \tau} d \tau\right| \leq c_{2}$. From (43), we have

$$
\left|e_{m 1}\right| \leq\left\|\underline{e}_{m}\right\| \leq \sqrt{\frac{2 \alpha}{\lambda_{\min }(P)}} \sqrt{\frac{\pi}{\mu}+\left(V(0)-\frac{\pi}{\mu}\right) e^{-\mu t}+c_{2}} .
$$

Let $c_{3} \geq\left|e_{a 1}\right|$ and $c_{4}$ be the upper bound of $\left|\hat{e}_{1}\right|$. Then, from (45), we get the upper bound of $\left|e_{1}\right|$ as follows:

$$
\begin{aligned}
\left|e_{1}\right| & \leq\left|\tilde{e}_{1}\right|+\left|\hat{e}_{1}\right| \\
& \leq\left|e_{m 1}\right|+\left|e_{a 1}\right|+\left|\hat{e}_{1}\right| \\
& \leq c_{4}+c_{3}+\sqrt{\frac{2 \alpha}{\lambda_{\min }(P)}} \sqrt{\frac{\pi}{\mu}+c_{2}} \\
& \leq \kappa,
\end{aligned}
$$

where $\kappa=c_{4}+c_{3}+\sqrt{2 \alpha / \lambda_{\min }(P)} \sqrt{\pi / \mu+c_{2}}$. This ends the proof.

Remark 5. Note that an observer-based fuzzy adaptive controller for nonlinear multivariable systems with unknown control direction has been also investigated in [18]. The stability of the closed-loop system has been proven by using the SPR condition and the Lyapunov theory. A comparison between our paper and that of Liu et al. [18] is summarized in Table 1. 
Table 1

Comparison between our control scheme and that proposed in [18].

\begin{tabular}{|c|c|c|}
\hline Comparison & Our paper & Paper of Liu et al. (i.e. [18]) \\
\hline The class of the systems considered & $\begin{array}{l}\text { SISO nonlinear system (but, one can effort- } \\
\text { lessly extend our results to the class consid- } \\
\text { ered in [18]) }\end{array}$ & $\begin{array}{l}\text { MIMO nonlinear system (but each subsys- } \\
\text { tem can be considered as a SISO system with } \\
\text { bounded interconnection nonlinearities) }\end{array}$ \\
\hline The control gain & $\begin{array}{l}\text { It is a function of state-variables, but bounded } \\
\text { by a constant }\end{array}$ & It is constant \\
\hline Number of design parameters & $\begin{array}{l}\text { There are nine design parameters to be deter- } \\
\text { mined }\end{array}$ & $\begin{array}{l}\text { There are three design parameters (for each } \\
\text { subsystems) to be determined }\end{array}$ \\
\hline Filtering of the fuzzy basis functions (FBF) & It is not necessary & It is necessary \\
\hline Number of the parameters to be adjusted & $\begin{array}{l}\text { There are } m+3 \text { parameters to be adjusted, } \\
\text { where } m \text { is the number of the fuzzy rules }\end{array}$ & There are three parameters to be adjusted \\
\hline Conclusions & $\begin{array}{l}\text { The advantages of our control scheme are: } \\
1 \text {. The class of the systems considered is rela- } \\
\text { tively large, as the control gain is not constant } \\
2 \text {. Because the FBFs are not filtered, the com- } \\
\text { putation burden is relatively reduced }\end{array}$ & $\begin{array}{l}\text { The advantages of the controller proposed in } \\
\text { [18]: } \\
\text { 1. There are fewer parameters to be adapted. } \\
\text { Therefore, there are little design parameters } \\
\text { to be determined } \\
\text { 2. Despite the FBF's filtering, the controller } \\
\text { proposed in [18] is simple. And because there } \\
\text { are fewer design parameters to be determined, } \\
\text { it is more appropriate in the practical applica- } \\
\text { tions }\end{array}$ \\
\hline
\end{tabular}

\section{Simulation results}

Simulation studies are carried out to show the effectiveness of the proposed controller. Two control problems are considered to this end. The first one concerns a Duffing oscillator, while the second one concerns an inverted pendulum system.

\subsection{Example 1}

In the following, we present simulation results showing the performances of the proposed fuzzy adaptive controller applied to a Duffing oscillator.

The Duffing equation describes the hardening spring effect observed in many mechanical systems or pendulum moving in a viscous medium. This chaotic system can be described as [7]

$$
\left\{\begin{array}{l}
\dot{x}_{1}=x_{2}, \\
\dot{x}_{2}=-p_{1} x_{2}-p_{2} x_{1}-p_{3} x_{1}^{3}+q \cos (\omega t),
\end{array}\right.
$$

where $\underline{x}=[x, \dot{x}]^{T}=\left[x_{1}, x_{2}\right]^{T}$ is the state vector, $p_{1}, p_{2}, p_{3}$, and $q$ are positive constants, $t$ is the time variable, and $\omega$ is the frequency. Depending on the choice of these constants, it is known that the solutions of (47) exhibit periodic, almost periodic, and chaotic behaviour [7]. A typical chaotic behaviour of the uncontrolled Duffing equation can be obtained with

$$
p_{1}=0.4, \quad p_{2}=-1.1, \quad p_{3}=1, \quad q=2.1 \quad \text { and } \omega=1.8 .
$$

Then, the controlled Duffing equation can be written as follows:

$$
\begin{aligned}
& \underline{\dot{x}}=\left[\begin{array}{ll}
0 & 1 \\
0 & 0
\end{array}\right] \underline{x}+\left[\begin{array}{l}
0 \\
1
\end{array}\right](f(\underline{x})+g(\underline{x}) u+d(t)), \\
& y=\left[\begin{array}{ll}
1 & 0
\end{array} \underline{x},\right.
\end{aligned}
$$


where $f(\underline{x})=-p_{1} x_{2}-p_{2} x_{1}-p_{3} x_{1}^{3}+q \cos (\omega t)$ and $g(\underline{x})=1$. It is assumed that the external disturbance $d(t)$ is a square wave having an amplitude \pm 1 with a period of $2 \pi(s)$.

The control objective is to force the system output $y$ to track the reference signal $y_{r}=\sin (t)$. It is worth noting that the function $f(\underline{x})$ and the control gain $g(\underline{x})$ are assumed here to be unknown by the controller and only the system output $y$ is measurable. In fact, the model (48) is only required for simulation purposes.

The observer-gain vector and the feedback-gain vector are selected respectively as follows: $K_{o}=[40,400]^{T}$ and $K_{c}^{T}=[4,4]$. We must choose the SPR filter $T(s)$ so that $\bar{H}(s)=H(s) T^{-1}(s)=\left(1 /\left(s^{2}+44 s+564\right)\right) T^{-1}(s)$ is also SPR. The filter $T(s)$ is selected as follows:

$$
T(s)=\frac{1}{s+8.2383} \text {. }
$$

From the expression of $\bar{H}(s)$, we can find that

$$
\bar{A}=\left[\begin{array}{cc}
-44 & 1 \\
-564 & 0
\end{array}\right], \quad \bar{B}^{T}=\left[\begin{array}{ll}
1 & 8.2383
\end{array}\right], \quad \bar{C}^{T}=\left[\begin{array}{ll}
1 & 0
\end{array}\right], \quad \text { and } \quad \alpha=0.4636 .
$$

Given

$$
Q=\left[\begin{array}{rr}
12 & -1 \\
-1 & 0.5
\end{array}\right]
$$

solving the matrix equation (33), we obtain the following positive-definite matrix:

$$
P=\left[\begin{array}{cc}
1.8496 & -0.1683 \\
-0.1683 & 0.0204
\end{array}\right] \text {. }
$$

The design parameters are selected as: $\gamma_{1}=500, \gamma_{2}=20, \gamma_{3}=4, \sigma_{1}=10^{-4}, \sigma_{2}=10^{-5}, \sigma_{3}=10^{-4}, \varepsilon_{1}=0.0125$ and $\varepsilon_{2}=0.1$.

For each variable of the inputs of the adaptive fuzzy system $\theta^{T} \psi\left(\underline{\hat{x}}_{v}\right)$, as in [1], we define three (triangular and trapezoidal) membership functions uniformly distributed on the intervals $[-2,2]$ for $\hat{x}_{1}$ and $[-3,3]$ for $\hat{x}_{2},[-5,5]$ for $\hat{v}$.

The initial conditions are chosen as $\underline{x}(0)=\left[x_{1}(0), x_{2}(0)\right]^{T}=[1,0]^{T}, \underline{\hat{e}}(0)=\left[\hat{e}_{1}(0), \hat{e}_{2}(0)\right]^{T}=[2,-2]^{T}, \rho(0)=10$, $\lambda(0)=0.5$ and $\theta_{i}(0)=0$.

Fig. 3 shows the simulation results obtained by applying the proposed fuzzy adaptive controller based on observer. Fig. 3(a) and (b) illustrates the tracking performances of the state variables. The estimates of tracking errors $\left(\hat{e}_{1}\right.$ and $\hat{e}_{2}$ ) are given in Fig. 3(c). Fig. 3(d) shows the boundedness of the control input $u$.

\subsection{Example 2}

In this section, we present simulation results showing the tracking performances of the proposed fuzzy adaptive controller applied to an inverted pendulum system.

Let $x_{1}=\theta$ be the angle of the pendulum with respect to the vertical line and $x_{2}=\dot{\theta}$. The dynamic equations of such a system are given by [30]

$$
\begin{aligned}
& {\left[\begin{array}{l}
\dot{x}_{1} \\
\dot{x}_{2}
\end{array}\right]=\left[\begin{array}{ll}
0 & 1 \\
0 & 0
\end{array}\right]\left[\begin{array}{l}
x_{1} \\
x_{2}
\end{array}\right]+\left[\begin{array}{l}
0 \\
1
\end{array}\right]\left(f\left(x_{1}, x_{2}\right)+g\left(x_{1}, x_{2}\right) u+d(t)\right),} \\
& y=\left[\begin{array}{ll}
1 & 0
\end{array}\right]\left[\begin{array}{l}
x_{1} \\
x_{2}
\end{array}\right] .
\end{aligned}
$$

with

$$
f\left(x_{1}, x_{2}\right)=\frac{m l x_{2} \sin x_{1} \cos x_{1}-(M+m) G \sin x_{1}}{m l \cos ^{2} x_{1}-\frac{4}{3} l(M+m)}, \quad g\left(x_{1}, x_{2}\right)=\frac{-\cos x_{1}}{m l \cos ^{2} x_{1}-\frac{4}{3} l(M+m)},
$$



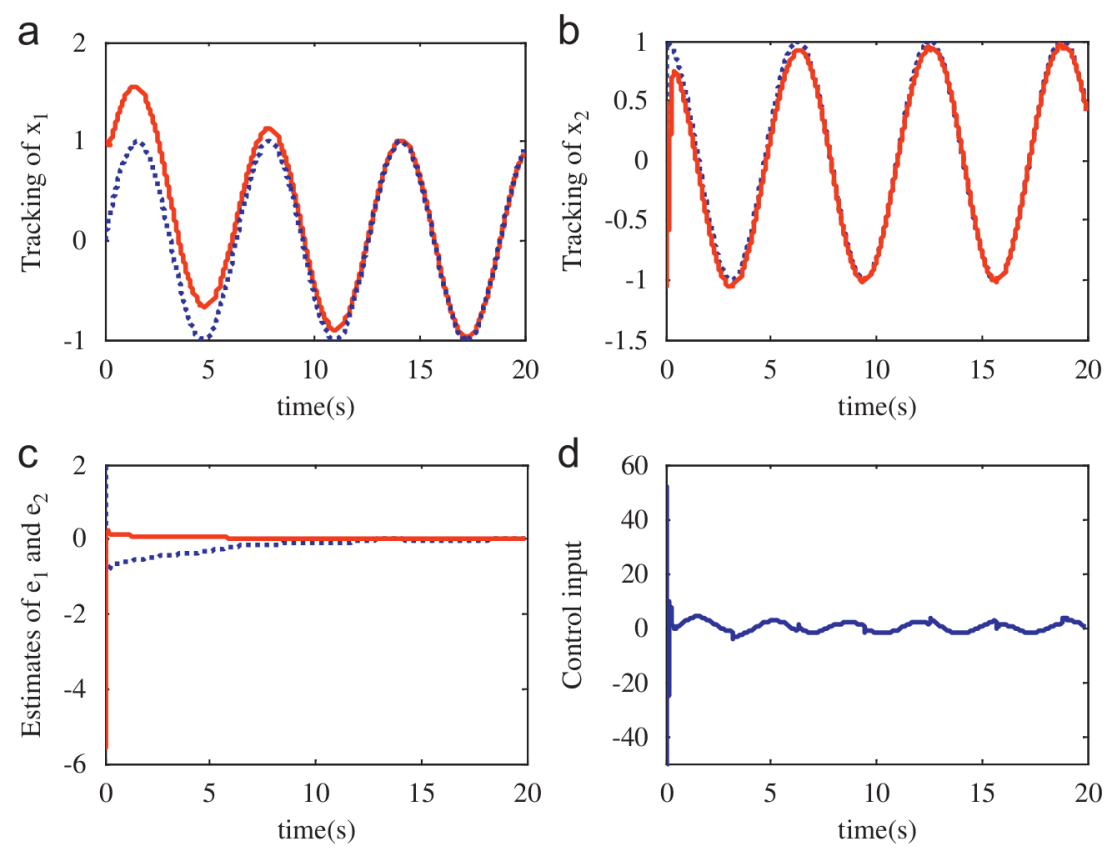

Fig. 3. Simulation results of Example 1: (a) the system output $y=x_{1}$ (solid line) and the reference signal $y_{r}$ (doted line). (b) The state variable $x_{2}$ (solid line) and the reference signal $\dot{y}_{r}$ (doted line). (c) The tracking error estimates $\hat{e}_{1}$ (doted line) and $\hat{e}_{2}$ (solid line). (d) The control input $u$.

where $G$ is the acceleration due gravity, $M$ is the mass of the cart, $m$ is the mass of the pole, $l$ is the half-length of pole and $u$ is the applied force.

It is assumed that the external disturbance $d(t)$ is a square wave having an amplitude \pm 1 with a period of $2 \pi(s)$. The system parameters are given as $M=1 \mathrm{~kg}, m=0.1 \mathrm{~kg}, l=0.5 \mathrm{~m}, G=9.8 \mathrm{~m} / \mathrm{s}^{2}$.

The control objective is to force the system output $y$ to track the reference signal $y_{r}=\sin (t)$. We assume that the functions $f(\underline{x})$ and $g(x)$ are completely unknown by the controller (including the sign of $g(x)$ ) and only the system output $y$ is available for measurement. In fact, the model (49) is only required for simulation purposes.

The observer-gain vector and the feedback-gain vector are selected respectively as follows: $K_{o}=[40,400]^{T}$ and $K_{c}^{T}=[24,144]$.

The SPR filter $T(s)$ is designed as follows:

$$
T(s)=\frac{1}{s+4.7787} \text { thus } \bar{H}(s)=H(s) T^{-1}(s)=\frac{s+4.7787}{s^{2}+184 s+6184} .
$$

From the expression of $\bar{H}(s)$, we can find that

$$
\bar{A}=\left[\begin{array}{cc}
-184 & 1 \\
-6184 & 0
\end{array}\right], \quad \bar{B}^{T}=\left[\begin{array}{ll}
1 & 4.7787
\end{array}\right], \quad \bar{C}^{T}=\left[\begin{array}{ll}
1 & 0
\end{array}\right], \quad \text { and } \alpha=0.1967 .
$$

Given $Q=\left[\begin{array}{cc}12 & -1 \\ -1 & 0.5\end{array}\right]$ solving the matrix equation (33), we get the following positive definite matrix:

$$
P=\left[\begin{array}{cc}
0.2351 & -0.008 \\
-0.008 & 0.0017
\end{array}\right] \text {. }
$$

The design parameters are selected as: $\gamma_{1}=500, \gamma_{2}=20, \gamma_{3}=0.25, \sigma_{1}=10^{-4}, \sigma_{2}=10^{-5}, \sigma_{3}=0.004$, $\varepsilon_{1}=0.0125$ and $\varepsilon_{2}=0.1$.

For each variable of the inputs of the adaptive fuzzy system $\theta^{T} \psi\left(\underline{\hat{x}}_{v}\right)$, as in [1], we define three (triangular and trapezoidal) membership functions uniformly distributed on the intervals $[-2,2]$ for $\hat{x}_{1},[-3,3]$ for $\hat{x}_{2}$ and $[-5,5]$ for $\hat{v}$. 

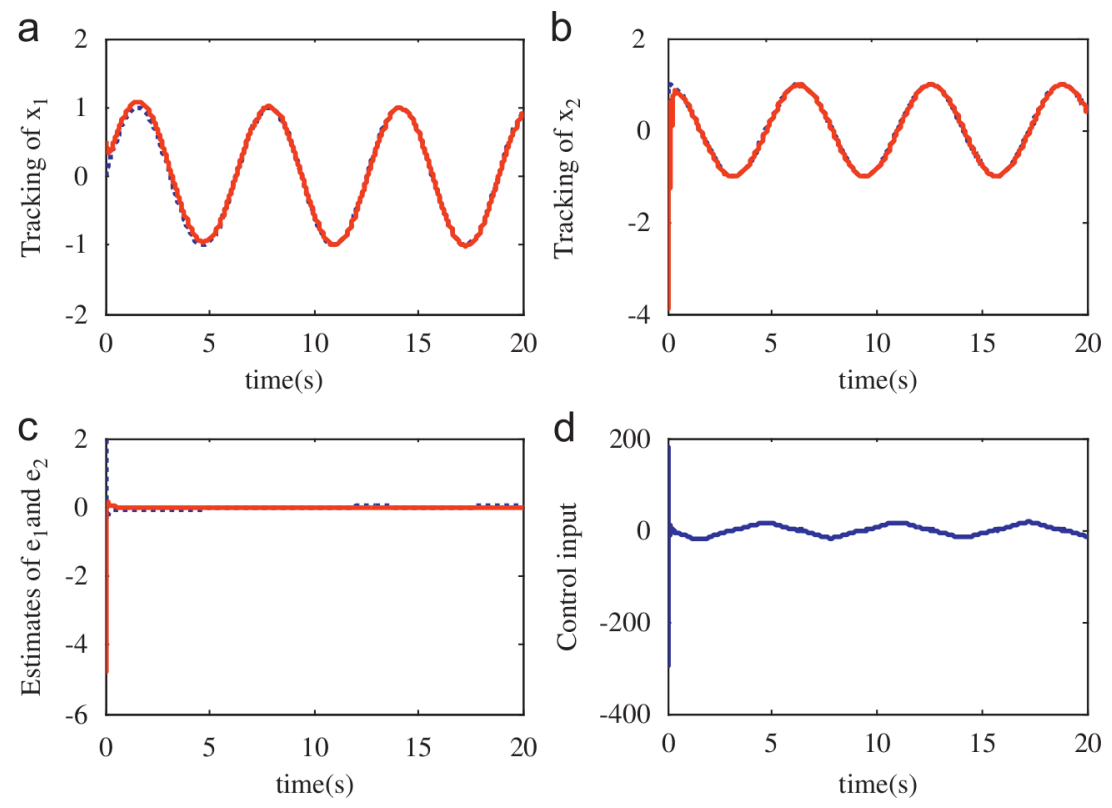

Fig. 4. Simulation results of Example 2: (a) the system output $y=x_{1}$ (solid line) and the reference signal $y_{r}$ (doted line). (b) The state variable $x_{2}$ (solid line) and the reference signal $\dot{y}_{r}$ (doted line). (c) The tracking error estimates $\hat{e}_{1}$ (doted line) and $\hat{e}_{2}$ (solid line). (d) The control input $u$.

The initial conditions are chosen as $\underline{x}(0)=\left[x_{1}(0), x_{2}(0)\right]^{T}=[0.5,0]^{T}, \underline{\hat{e}}(0)=\left[\hat{e}_{1}(0), \hat{e}_{2}(0)\right]^{T}=[2,-2]^{T}$, $\rho(0)=10, \lambda(0)=0.5$ and $\theta_{i}(0)=0$.

The simulation results are depicted in Fig. 4. From this figure, we can see that the system tracks its desired trajectories and the control input is bounded.

\section{Conclusion}

In this paper, an observer-based fuzzy adaptive controller for a class of SISO nonlinear systems with unknown control gain sign has been presented. In the controller designing, neither measurement of the system states nor knowledge of the system nonlinearities are required. Indeed, an observer has been constructed to estimate the tracking error vector and an adaptive fuzzy system has been used to approximate the system nonlinearities. The Nussbaum-type function has been particularly used to deal with the unknown control gain sign. Using the SPR condition and Lyapunov theory, the stability of the closed-loop system has been rigorously proven. Simulation results have been reported to emphasize the performances of the proposed controller.

\section{References}

[1] A. Boulkroune, M. Tadjine, M. M'saad, M. Farza, How to design a fuzzy adaptive control based on observers for uncertain affine nonlinear systems, Fuzzy Sets Syst. 159 (2008) 926-948.

[2] A. Boulkroune, M. Tadjine, M. M'saad, M. Farza, Adaptive fuzzy controller for non-affine systems with zero dynamics, Int. J. Syst. Sci. 40 (4) (2009) 367-382.

[3] A. Boulkroune, M. M’Saad, M. Tadjine, M. Farza, Fuzzy adaptive controller for MIMO nonlinear systems with known and unknown control direction, Fuzzy Sets Syst. 161 (2010) 797-820.

[4] A. Boulkroune, M. M'Saad, H. Chekireb, Design of a fuzzy adaptive controller for MIMO nonlinear time-delay systems with unknown actuator nonlinearities and unknown control direction, Inf. Sci. 180 (2010) 5041-5059.

[5] A. Boulkroune, M. M'Saad, M. Farza, Adaptive fuzzy controller for multivariable nonlinear state time-varying delay systems subject to input nonlinearities, Fuzzy Sets Syst. 164 (2011) 45-65.

[6] Y.C. Chang, Adaptive fuzzy-based tracking control for nonlinear SISO systems via VSS and $H^{\infty}$ approaches, IEEE Trans. Fuzzy Syst. 9 (2001) 278-292.

[7] G. Chen, X. Dong, On feedback control of chaotic continuous-time systems, IEEE Trans. Circuits Syst. I 40 (1993) 591-600. 
[8] B.S. Chen, C.H. Lee, Y.C. Chang, $H^{\infty}$ Tracking design of uncertain nonlinear SISO systems: adaptive fuzzy approach, IEEE Trans. Fuzzy Syst. 4 (1) (1996) 32-43.

[9] M. French, E. Rogers, Approximate models for adaptive feedback linearization, Int. J. Control 68 (6) (1997) 1305-1321.

[10] S.S. Ge, F. Hong, T.H. Lee, Adaptive neural control of nonlinear time-delay systems with unknown virtual control coefficients, IEEE Trans. Syst. Man Cybern. Part B Cybern. 34 (2004) 499-516.

[11] F. Hong, S.S. Ge, B. Ren, T.H. Lee, Robust adaptive control for a class of uncertain strict-feedback nonlinear systems, Int. J. Robust Nonlinear Control 19 (2009) 746-767.

[12] K. Hornik, Multilayer feedforward networks are universal approximators, Neural Networks 2 (1989) 359-366.

[13] N. Kim, A.J. Calise, Several extensions in methods for adaptive output feedback control, IEEE Trans. Neural Networks 18 (2) (2007) $482-494$.

[14] Y.G. Leu, T.T. Lee, W.Y. Wang, Observer-based adaptive fuzzy-neural control for unknown nonlinear dynamical systems, IEEE Trans. Syst. Man Cybern. Part B Cybern. 29 (1999) 583-591.

[15] Y.G. Leu, W.Y. Wang, T.T. Lee, Observer-based direct adaptive fuzzy-neural control for nonaffine nonlinear systems, IEEE Trans. Neural Networks 16 (4) (2005) 853-861.

[16] H.X. Li, S. Tong, A hybrid fuzzy adaptive control for a class of nonlinear MIMO systems, IEEE Trans. Fuzzy Syst. 11 (1) (2003) $24-34$.

[17] Y.J. Liu, S.C. Tong, W. Wang, Y.M. Li, Observer-based direct adaptive fuzzy control of uncertain nonlinear systems and its applications, Int. J. Control Autom. Syst. 7 (4) (2009) 681-690.

[18] Y.J. Liu, S.C. Tong, T.S. Li, Observer-based adaptive fuzzy tracking control for a class of uncertain nonlinear MIMO systems, Fuzzy Sets Syst. 164 (1) (2011) 25-44.

[19] R.D. Nussbaum, Some remarks on the conjecture in parameter adaptive control, Syst. Control Lett. 3 (5) (1983) $243-246$.

[20] J.H. Park, G.T. Park, S.H. Kim, C.J. Moon, Output feedback control of uncertain nonlinear systems using a self-structuring adaptive fuzzy observer, Fuzzy Sets Syst. 151 (2005) 21-42.

[21] M.M. Polycarpou, P.A. Ioannou, A robust adaptive nonlinear control design, Automatica 32 (3) (1996) $423-427$.

[22] E.P. Ryan, A universal adaptive stabilizer for a class of nonlinear systems, Syst. Control Lett. 16 (1991) 209-218.

[23] S. Sastry, A. Isidori, Adaptive control of linearizable systems, IEEE Trans. Autom. Control 34 (11) (1989) 1123-1131.

[24] J.E. Slotine, W. Li, Applied Nonlinear Control, Prentice-Hall, Englewood Cliffs, NJ, 1991.

[25] J.T. Spooner, K.V. Passino, Stable adaptive control using fuzzy systems and neural networks, IEEE Trans. Fuzzy Syst. 4 (3) (1996) $339-359$.

[26] C.Y. Sue, Y. Stepanenko, Adaptive control of a class of nonlinear systems with fuzzy logic, IEEE Trans. Fuzzy Syst. 2 (4) (1994) $285-294$.

[27] S. Tong, H.X. Li, W. Wang, Observer-based adaptive fuzzy control for SISO nonlinear systems, Fuzzy Sets Syst. 148 (2004) $355-376$.

[28] S. Tong, H.X. Li, G.R. Chen, Adaptive fuzzy decentralized control for a class of large-scale nonlinear systems, IEEE Trans. Syst. Man Cybern. Part B Cybern. 134 (1) (2004) 770-775.

[29] L.X. Wang, J.M. Mendel, Fuzzy basis functions, universal approximation, and orthogonal least square learning, IEEE Trans. Neural Networks 3 (5) (1992) 807-814.

[30] L.X. Wang, Adaptive Fuzzy Systems and Control: Design and Stability Analysis, Prentice-Hall, Englewood Cliffs, NJ, 1994.

[31] W.Y. Wang, Y.G. Leu, T.T. Lee, Output-feedback control of nonlinear systems using direct adaptive fuzzy-neural controller, Fuzzy Sets Syst. 140 (2003) 341-358.

[32] X.D. Ye, Adaptive nonlinear output-feedback control with unknown high-frequency gain sign, IEEE Trans. Autom. Control 46 (1) (2001) $112-115$. 